\title{
A Novel Process Model of Ship Rust Removal by Premixed Abrasive Jet based on Neural Network
}

\author{
Qing Guo ${ }^{1}$, Shuzhen Yang ${ }^{1,2}$ and Tao $\mathrm{Yu}^{1}$ \\ ${ }^{1}$ School of Mechatronic Engineering and Automation, Shanghai University, Shanghai, China \\ ${ }^{2}$ School of Intelligent Manufacturing and Control Engineering, Shanghai Polytechnic University, Shanghai, China
}

\begin{abstract}
In view of the technological requirements of the development of green shipbuilding technology on the effect of ship surface rust removal, the premixed abrasive jet technology is used to remove rust. Because the rust removal of ships with premixed abrasive jet is influenced by multiple parameters and has a high nonlinear relationship between various parameters, the accurate process model of it is difficult to establish. On the basis of artificial neural network modelling technology, the model of ship rust removal with premixed abrasive jet is built. The model takes the system pressure, the target distance, the moving speed of the spray gun and the particle size of the abrasive as input parameters, and the score which can most reflect the effect of the rust removal as output parameter. The test results show that the prediction error of the model is small, and it can better reflect the process rule between the effect of the premixed abrasive jet and the process parameters. We can guide the selection of process parameters according to the model.
\end{abstract}

\section{Introduction}

Due to the special working conditions of high salinity and high humidity, the corrosion degree of the surface of the ship is generally serious and the corrosion area is large. It is very difficult to effectively remove rust on its surface, and not pollute the river where the ship is located at the same time [1]. The premixed abrasive jet technology is widely concerned because of its high rust removal efficiency, no pollution and low equipment requirements [2]. Its abrasive particles and water medium are mixed evenly, and the quality of jet is superior to that of other jets [3]. But so far, the setting of the parameters before processing depends on the experience of the rust removal workers and the best parameters obtained by the single factor experiment [4-5], which ignores the coupling effect between the various process parameters and is not universal.

Artificial neural network is a complex network system which has the characteristics of flexible self-learning, self-organization and self-adapting. Through network learning, it can approximate any linear or nonlinear functions [6]. In this paper, artificial neural network modelling technology is used to build the model of ship rust removal with premixed abrasive jet. The effect of the ship rust removal is predicted based on the established processing parameters, and the model is used to guide the selection of the processing parameters conversely.

\section{The principle of ship rust removal by premixed abrasive jet}

The principle of the ship rust removal device is shown in Figure 1. In order to ensure the continuous feeding of the ship rust removal system of premixed abrasive jet, the working mode of the double storage tank is used alternately. Before work, the mud pump and control valves are used for feeding. During the work, the water source is added to the abrasive tank. At the same time, it is injected into the mixing chamber too, so that the liquidsolid two phase flow which flows into the mixing chamber from the abrasive tank is mixed fully. Finally, through the acceleration of the pipeline, the abrasive is sent to the spray nozzle on the surface of the ship's steel plate to carry out the ship rust removal operation. With the kinetic energy of high pressure water, the abrasive particles are constantly impacting and grinding the hull on the surface of the hull, and the quality and efficiency of the jet are high. Experiments and the collection of sample data in this paper is based on the equipment which is independently developed. Considering the factors such as hardness, strength, toughness, rust removal effect, rust removal efficiency and cost, the ship rust removal device decides to use river sand that does not cause environmental pollution in the river as abrasive particles. 


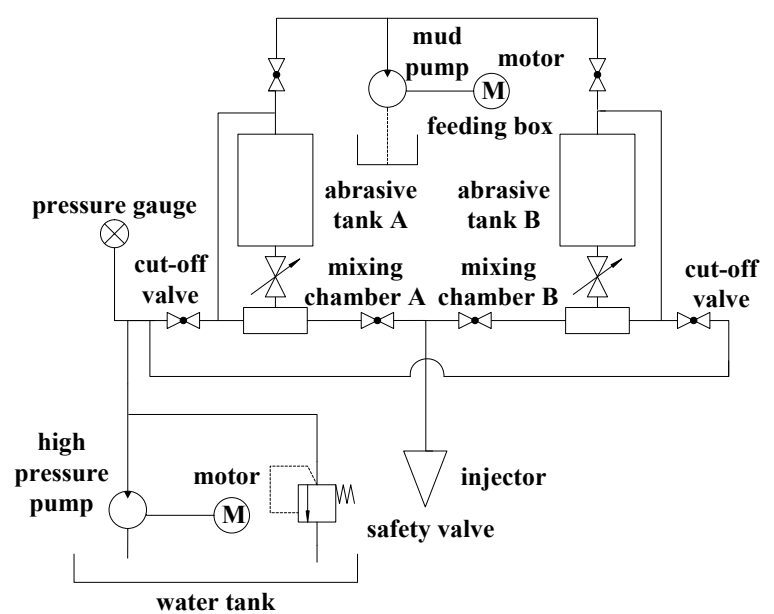

Figure 1. Principle of the ship rust removal device.

\section{Process model of rust removal by premixed abrasive jet}

\subsection{Determination of the parameters of the input layer / output layer}

There are many factors affecting the process of rust removal, such as pressure, flow, abrasive, nozzle, injection angle, jet target distance, jet speed and so on. The effect of rust removal is the result of the common effect of these factors. In order to simplify the model, combining the experience of the rust removal equipment operators and related information, finally, it is determined that the input parameters of the model are system pressure, target distance, moving speed of spray gun and abrasive particle size [7].

$\mathrm{Sa}$ is used to represent the effect of the jet injection on the rust removal in general, which is divided into 4 grades of $\mathrm{Sa} 1, \mathrm{Sa} 2, \mathrm{Sa} 2.5$ and $\mathrm{Sa} 3$. In order to quantify the effect of rust removal, corresponding the above 4 grades to a different score, as shown in Table 1. The model is established by using the score that can most reflect the effect of rust removal as the output parameter of the model.

Table 1. Evaluation standard of rust removal effect.

\begin{tabular}{lll}
\hline Grade & Score & Evaluation criterion \\
\hline Sa1 & 1 & $\begin{array}{l}\text { There is no visible oil, dirt and insecure } \\
\text { attachment on the surface of the steel } \\
\text { plate. } \\
\text { There is no visible oil or dirt on the } \\
\text { surface of the steel plate. Basically } \\
\text { scavenging the oxide skin, rust and } \\
\text { other attachments and has strong } \\
\text { adherent residue. }\end{array}$ \\
Sa2 & 3 & $\begin{array}{l}\text { There is no visible oil, dirt, and oxide, } \\
\text { rust, paint and other attachments on the } \\
\text { Surface of the steel plate. Only trace or } \\
\text { striped spots are retained. } \\
\text { The surface of the steel plate has no } \\
\text { visible oil, dirt, and oxide, rust, paint } \\
\text { and other attachments, with a uniform } \\
\text { metallic lustre. }\end{array}$ \\
Sa3 & 4 & 5
\end{tabular}

\subsection{Determination of model structure}

There are many types of neural networks. BP neural network is a kind of artificial neural network which uses error back propagation. It is the most widely used. In this paper, based on BP neural network, the process model of ship rust removal with premixed abrasive jet is constructed. The specific formula is as follows:

$$
\mathrm{y}=\mathrm{g}\left(\sum_{j=1}^{m}\left(W_{j} f\left(\sum_{i=1}^{n} V_{i j} X_{i}+b_{j}\right)+b\right)\right)
$$

Where $\mathrm{x}$ represents input vector, $\mathrm{y}$ represents output vector, $i$ represents the number of variables in the input layer, in this article is $4, \mathrm{j}$ represents the number of neurons in the hidden layer, $V_{i j}$ represents the weight matrix between the input layer and the hidden layer, $W_{j}$ represents the weight matrix between the hidden layer and the output layer, $b$ and $b_{j}$ represent neural network threshold, $f$ and $g$ represent transfer function and activation function. In this paper, the sigmoid tangent hyperbolic tangent function and the purelin linear transformation function are used as the transfer function and the activation function.

In neural networks, an appropriate increase in the number of hidden layers can improve the learning and generalization ability of the model. But the error signal is transmitted from the hidden layer to the input layer by the output layer. The more hidden layers, the more unreliable when the error passes to the input layer. Referring to related literature [8], we can see that a single hidden layer neural network with enough neurons can approximate any function which is smooth, measurable between input and output by selecting appropriate connection weights and transfer functions. In order to simplify the model, this paper decides to adopt one hidden layer. The structure of BP neural network for ship rust removal with premixed abrasive jet as shown in Figure 2.

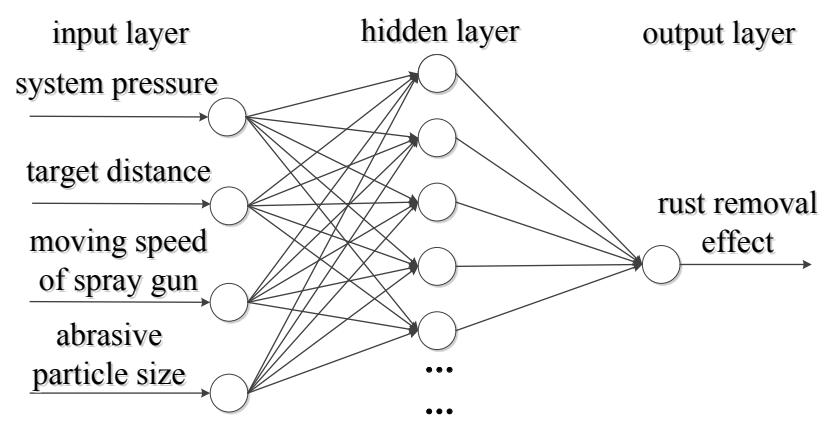

Figure 2. Network structure of BP artificial neural.

\subsection{Algorithm and structure optimization}

The BP neural network itself has some shortcomings, which is mainly reflected in the slow convergence of the learning process and easy to fall into local minimum. In view of the above defects, many scholars have proposed different improved algorithms. In this paper, the L-M algorithm (Levenberg-Marquardt) is used to improve the stability of the model. L-M algorithm adopts numerical optimization technology, combines the advantages of gradient descent method and Gauss Newton method. It 
can not only improve the convergence speed of network, but also reduce the possibility of falling into local minimum.

The number of neurons in the hidden layer will directly affect the accuracy and generalization of the model. If the number of neurons is too small, it will lead to lack of fitting, and the network can't describe the data accurately. If the number of neurons is too large, it will lead to excessive fitting and affect its performance. The number of neurons can be determined by comparing the mean relative error of different neuron numbers, it can also be determined by the empirical formula of hidden layer neurons:

$$
\mathrm{M}=(N+P)^{1 / 2}+c
$$

Where $\mathrm{c}$ takes the integers between 1 and $10, \mathrm{~N}$ represents the number of neurons in the input layer, in this paper is 4 , $\mathrm{P}$ represents the number of neurons in the output layer, in this paper is $1, \mathrm{M}$ represents the number of neurons in the hidden layer, takes the integers between 3 and 13.

\section{Experiments}

\subsection{Acquisition and pre-processing of sample data}

There are many factors affecting the effect of rust removal. In order to eliminate the influence of other factors on the network, it is necessary to ensure the consistency of the other factors in each experiment. Through preliminary experiments, it is found that the coupling effect between the injection angle and the other factors is smaller. Refer to the relevant literature, and the verification of the single factor experiment, it is found that the ship's rust removal effect is the best when the angle of the jet and the corroded steel plate is 60 degrees [9]. The relevant parameters of the experiment are set as follows:

(1) System pressure. According to the preliminary experiment, the effect of rust removal is better when the pressure of the system is over $7 \mathrm{MPa}$. However, if the pressure is too large, the gun recoil will increase quickly, the gunman is difficult to grasp which is danger. Therefore, the initial system pressure is between 7 and 11 $\mathrm{MPa}$.

(2) Target distance. The target distance refers to the distance between the jet nozzle and the surface of the ship's steel plate, it is best to be fixed in the area of the energy concentration of the jet. If the target distance is too large, it will lose more power and energy of the jet, and can't play a very good damage to the surface of the ship. The initial target distance is from $300 \mathrm{~mm}$ to $500 \mathrm{~mm}$.

(3) Moving speed of the spray gun. When the moving speed of the spray gun is too small, it can better remove the rust and the attachment, but the efficiency is not high. In order to ensure the rust removal efficiency, the initial moving speed of the spray gun is $300 \sim 700 \mathrm{~mm} / \mathrm{min}$.

(4) Abrasive particle size. The particle size of the abrasive has a certain influence on the quality and efficiency of the rust removal. According to the experience of the operators and preliminary experiments, mesh number is taken for the 16, 18, 20, 24 and 28 .

In summary, part of the sample data is shown in Table 2. Because neural network is sensitive to sample data, the order of sample data and the difference between numerical values of different parameters will affect the training time and accuracy. Practice has proved that in the process of network training, we first normalize sample data and import them into neural network for training, which can improve the efficiency, accuracy and generalization ability of network training. The premnmx function is used to normalize the sample data.

\subsection{Results and analysis}

Based on the MATLAB R2015b software platform, the functions of newff and train in the artificial neural network toolbox are called, the training and testing of the network are carried out. Training with the first 40 groups of data, then testing with the last 10 groups of data. The relationship between the number of neurons in the hidden layer and the average relative error of the prediction results is shown in Table 3. It is known that when the number of neurons is 8 , the average relative error is the smallest, which is $1.34 \%$. Therefore, it is decided to adopt the network structure of 4-8-1.

Table 2. Part of the sample data.

\begin{tabular}{cccccc}
\hline & $\begin{array}{c}\text { System } \\
\text { pressure(MPa) }\end{array}$ & $\begin{array}{c}\text { Target distance } \\
(\mathrm{mm})\end{array}$ & $\begin{array}{c}\text { Moving speed of the } \\
\text { spray gun }(\mathrm{mm} / \mathrm{min})\end{array}$ & $\begin{array}{c}\text { Abrasive particle size (mesh } \\
\text { number) }\end{array}$ & $\begin{array}{c}\text { Rust removal } \\
\text { effect }\end{array}$ \\
\hline 1 & 7 & 300 & 300 & 16 & 3.97 \\
2 & 7 & 350 & 400 & 18 & 4.01 \\
3 & 7 & 400 & 500 & 20 & 4.14 \\
4 & 7 & 450 & 600 & 24 & 3.86 \\
5 & 7 & 500 & 700 & 28 & 3.71 \\
6 & 7 & 300 & 400 & 24 & 3.94 \\
7 & 7 & 350 & 300 & 28 & 4.07 \\
8 & 7 & 400 & 600 & 16 & 3.76 \\
9 & 7 & 450 & 700 & 18 & 3.81 \\
10 & 7 & 500 & 500 & 20 & 4.11 \\
11 & 8 & 300 & 500 & 16 & 4.12 \\
$\ldots$ & & & & & 28 \\
50 & 11 & 500 & 300 & & 4.71 \\
\hline
\end{tabular}


Table 3. The relationship between the number of neurons in the hidden layer and the model error.

\begin{tabular}{cc}
\hline $\begin{array}{c}\text { The number of neurons in } \\
\text { the hidden layer }\end{array}$ & $\begin{array}{c}\text { Average relative error } \\
\text { of prediction }\end{array}$ \\
\hline 3 & $2.85 \%$ \\
4 & $1.92 \%$ \\
5 & $1.65 \%$ \\
6 & $2.91 \%$ \\
7 & $1.60 \%$ \\
8 & $1.34 \%$ \\
9 & $1.73 \%$ \\
10 & $2.84 \%$ \\
11 & $1.87 \%$ \\
12 & $1.68 \%$ \\
13 & $3.62 \%$ \\
\hline
\end{tabular}

The experiments were done to verify the effectiveness of the premixed abrasive jet ship rust removal process model. The processing parameters and the effect of rust removal were inputted into the model. The prediction results of the model were compared with the actual effect of rust removal. Figure 3 is the comparison curve of the effect of the actual rust removal and the prediction results of the model. The maximum error is $2.41 \%$, the minimum error is $0.03 \%$, and the average relative error is $1.32 \%$.

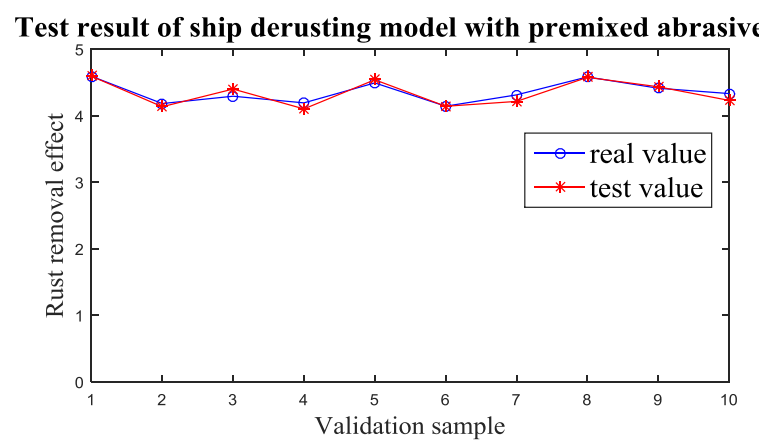

Figure 3. Test result of ship rust removal model with premixed abrasive jet.

Based on this model, taking a steel plate that is equivalent to the rust of the ship to test. After $243 \mathrm{~s}$, the corrosion of the steel plate was completely removed. The effect of rust removal reaches Sa2.5, and the efficiency of rust removal reaches $14 \mathrm{~m} 2 / \mathrm{h}$. Compared with the efficiency of rust removal not based on this model, it has increased by $16.7 \%$.

\section{Conclusion}

In view of the actual situation of the setting of processing parameters depends on the experience, the ship rust removal process model of premixed abrasive jet based on neural network is established. The prediction model can quickly predict the effect of rust removal when the system pressure, the target distance, the moving speed of the spray gun and the particle size of the abrasive are determined. The prediction model takes "4-8-1" as the optimal structure, with the maximum prediction error of $2.41 \%$, minimum prediction error of $0.06 \%$ and the average relative error of $1.34 \%$. In general, it can truly reflect the process rule between the effect of ship rust removal by premixed abrasive jet and the process parameters, we can guide the selection of process parameters based on this model.

\section{Acknowledgments}

This work was supported by Shanghai Committee of Science and Technology, China (Grant No.17511107302) and the Project of Key Discipline of Shanghai Polytechnic University (No. XXKZD1603).

\section{References}

1. Xie Yufei, Liu Hongwei, Hu Yongxiang. Study on the method of determining the technological parameters of laser rust removal of ship plate [J]. Chinese laser, 4, 103-110 (2016).

2. Liu Guoyong, Chen Xinxin, Zhu Dongmei, et al. Internal flow field of mixed abrasive jet mixing cavities of premixed abrasive jet [J]. Journal of University of Science and Technology Beijing, 4, 830-837 (2014).

3. Zuo Weiqin, Lu Yiyu, Liu Mingju, and so on. A new method of testing the abrasive speed of the premixed abrasive jet [J]. Journal of Chongqing University: Natural Science Edition, 39, 96-102 (2016).

4. Zhou Mengying. Application of premixed abrasive jet technology to pretreatment of anticorrosion of large metal components in coal mine [J]. Coal engineering, 1, 94-96 (2008).

5. Yang Gang. Simulation and study of abrasive water jet rust removal technology [J]. Mechanical and electrical engineering, 30, 929-932 (2013).

6. Liu H, Wang J, Kelson N, et al. A study of abrasive waterjet characteristics by CFD simulation [J]. Journal of Materials Processing Technology, 1, 488493 (2004).

7. Liu L H, Cao H, Zou C Q. System of Premixed Abrasive Jet Cleaning and Derusting on Steel Tube In wall [J]. Applied Mechanics \& Materials, 4, 25712574 (2012).

8. Shi Lingzhi, Deng Qihong, Lu Chan, et al. BP artificial neural network based on PM_atmospheric particles (10) concentration prediction [J]. Journal of Central South University (NATURAL SCIENCE EDITION), 43, 1969-1974 (2012).

9. Nguyen T, Shanmugam D K, Wang J. Effect of liquid properties on the stability of an abrasive waterjet[J]. International Journal of Machine Tools \& Manufacture, 48, 1138-1147 (2008). 